\section{Validação da terceira versão do Questionário Alimentar do Dia Anterior (QUADA-3) para escolares de 6 a 11 anos}

\author{
Validation of the third version of the Previous \\ Day Food Questionnaire (PDFQ-3) for \\ 6-to-11-years-old schoolchildren
}

\footnotetext{
${ }_{1}$ Programa de Pós-graduação em Nutrição, Universidade Federal de Santa Catarina Florianópolis, Brasil.

2 Programa de Pós-graduação em Saúde Pública

Universidade Federal de Santa Catarina Florianópolis, Brasil.

Correspondência M. A. A. Assis

Programa de Pós-graduação em Nutrição, Universidade Federal de Santa Catarina. Campus Universitário Trindade, Florianópolis, SC 88040-970, Brasil. massis@ccs.ufsc.br
}

\section{Abstract}

The aim of this study was to assess the validity of the third version of the Previous Day Food Questionnaire (PDFQ-3) for schoolchildren. PDFQ-3 is a structured instrument with 6 meals and 21 foods. The study included 164 students aged 6 to 11 years in a public school with a full-day school schedule in 2007. Validity was assessed by comparing food items selected on the questionnaire and direct observations of foods eaten in three school meals on the previous day (mid-morning snack, lunch, and mid-afternoon snack). Multivariate logistic regression analysis was used to evaluate the discordance between answers from the questionnaire versus those from observations. Results indicated high sensitivity and specificity for the majority of the food items. Mean values for sensitivity and specificity were $70.5 \%$ and $87.1 \%$, respectively, for 12 food items in three combined school meals. Schoolchildren aged 6 to 8 years showed higher discordance between PDFQ-3 and observations, compared to 9-to-11-year-olds. Based on the findings, the PDFQ-3 can generate valid data for the evaluation of previous day food consumption in groups of schoolchildren.

Food Consumption; Questionnaires; Child
Maria Alice Altenburg de Assis ${ }^{1}$

Jucemar Benedet 1

Raquel Kerpel 1

Francisco de Assis Guedes de Vasconcelos 1

Patrícia Faria Di Pietro ${ }^{1}$

Emil Kupek 2

\section{Introdução}

Nos últimos anos, vários estudos de validação foram realizados para testar técnicas e instrumentos de avaliação do consumo alimentar de crianças em idade escolar. Esses estudos, conduzidos para diagnosticar a acurácia das informações relatadas quando comparadas a um método de referência, fornecem informações importantes para o aprimoramento do formato e da linguagem do instrumento, protocolo de aplicação e verificação dos vieses relacionados às características da população-alvo 1 .

Questionários de consumo alimentar estruturados são métodos simples e alternativos ao inquérito recordatório e ao questionário de freqüência; apresentam maior praticidade e melhor custo-benefício para estudos epidemiológicos, bem como para avaliações de programas comunitários e escolares 2 . A observação direta de refeições escolares e o recordatório alimentar de 24 horas têm sido medidas de referência bastante utilizadas para testar técnicas de entrevistas ${ }^{3} \mathrm{e}$ validar questionários de consumo alimentar do dia anterior, destinados à mídia impressa 2,4,5,6 ou eletrônica 7,8,9.

No Brasil, são raros os instrumentos destinados à coleta de dados de consumo alimentar, que tenham sido validados para crianças em idade escolar. Para cobrir essa lacuna, foi desenvolvido o Questionário Alimentar do Dia Anterior (QUADA) 10,11,12,13. O QUADA é um instrumento ilustrado, 
delineado como um recordatório para obter dados de consumo de alimentos nas refeições do dia anterior para escolares. Esse questionário foi anteriormente submetido a dois estudos de validação, dentre uma série de testes propostos para diferentes contextos em futuras pesquisas. A primeira versão do instrumento, denominada Dia Típico de Atividade Física e de Consumo Alimentar (DAFA), foi testada com escolares de primeira a quarta série de uma escola pública, utilizandose como método de referência o recordatório de 24 horas 10. Na segunda versão (Questionário Alimentar do Dia Anterior), foi avaliada a reprodutibilidade e a validade, utilizando-se as observações diretas de refeições escolares como método de referência e optando-se pela definição do dia anterior para o período de consumo 12,13. Lições retiradas dos aspectos positivos e das limitações desses estudos 10,11,12,13 foram importantes para aprimorar a terceira versão do Questionário Alimentar do Dia Anterior (QUADA-3).

O objetivo deste artigo é apresentar os resultados do estudo de validação da QUADA-3 e avaliar a influência de variáveis relacionadas aos participantes do estudo sobre a discordância das respostas entre o questionário e as observações diretas de três refeições escolares.

\section{Métodos}

\section{Tipo de estudo e amostra}

Trata-se de um estudo de validação, com amostra de conveniência composta pela totalidade dos alunos do segundo ao quinto ano do Ensino Fundamental de uma escola pública do Balneário Camboriú, Estado de Santa Catarina, Brasil. A instituição foi escolhida por ser a única na região com atendimento em período integral, que fornecia três refeições diárias aos escolares (lanche da manhã, almoço e lanche da tarde). Dos 196 alunos convidados a participar do estudo, 185 tiveram o consentimento dos pais. Destes, foram excluídos os dados de 17 crianças que não estavam presentes no dia da observação e de quatro outras que, sendo portadoras de necessidades especiais, apresentavam dificuldade de resposta ao questionário.

\section{Classificação do estado nutricional}

Inicialmente, foram obtidos os dados da massa corporal e da estatura, seguindo-se os procedimentos recomendados pela Organização Mundial da Saúde (OMS) 14. A medida de massa corporal foi realizada com a utilização de balança digital da marca Filizola, com escalas de 100 gra- mas. Para mensuração da altura, utilizou-se uma fita métrica milimetrada, aderida à parede, com o ponto zero da fita aderida ao nível do solo. Para a obtenção desses dados, os escolares estavam descalços e usavam roupas leves. O índice de massa corporal (IMC) [massa corporal (kg)/estatura $\left(\mathrm{m}^{2}\right)$ ] foi utilizado para classificar as crianças em grupos de baixo peso e de excesso de peso, empregando-se os valores críticos do IMC para classificação do estado nutricional de crianças e adolescentes brasileiros 15 .

\section{Desenvolvimento do QUADA}

Detalhes do desenvolvimento das versões anteriores ao QUADA-3 foram descritos em outras publicações 10,11,12,13. O instrumento não foi delineado para obter informações sobre freqüências e quantidades, pois essas capacidades não estão bem desenvolvidas em crianças que ainda não alcançaram o estágio de raciocínio abstrato 16,17.

A versão QUADA-3, objeto do presente estudo, foi modificada em relação à versão QUADA-2 12 nos seguintes aspectos: (a) inclusão de mais uma refeição (após o jantar) para permitir uma aproximação ao período de 24 horas; (b) inclusão de mais cinco alimentos em cada refeição, com o intuito de servir como um estímulo à memória para o relato de alimentos importantes do ponto de vista nutricional; (c) modificação das ilustrações das crianças no QUADA-3, para tornar o formato do corpo mais adequado à faixa etária de 7 a 10 anos, uma vez que na versão QUADA-2 os desenhos eram mais parecidos com crianças pré-escolares.

O questionário está disposto em quatro páginas de papel formato A4. A primeira página contém espaço para identificação do escolar e ilustrações para escolha do tipo de transporte utilizado para ir à escola. As refeições foram ordenadas cronologicamente: café e lanche da manhã na segunda página, almoço e lanche da tarde na terceira página, jantar e lanche da noite na quarta página. Cada refeição foi ilustrada com 21 alimentos ou grupos de alimentos: bolacha salgada e pão; achocolatado com leite; café com leite; leite; iogurte; queijo; arroz; refrigerante; doces; salgadinho em pacote; batata frita; pizza e hambúrguer; frutas; feijão; macarrão; peixe e frutos do mar; carne de gado e frango; suco natural; hortaliças; sopa de verduras e verduras ( $\mathrm{Fi}$ gura 1). Os alimentos foram selecionados levando-se em consideração os padrões alimentares das crianças da faixa etária em observação, a disponibilidade de alimentos, o cardápio oferecido nas escolas públicas 18 e o Guia Alimentar para a População Brasileira 19. 


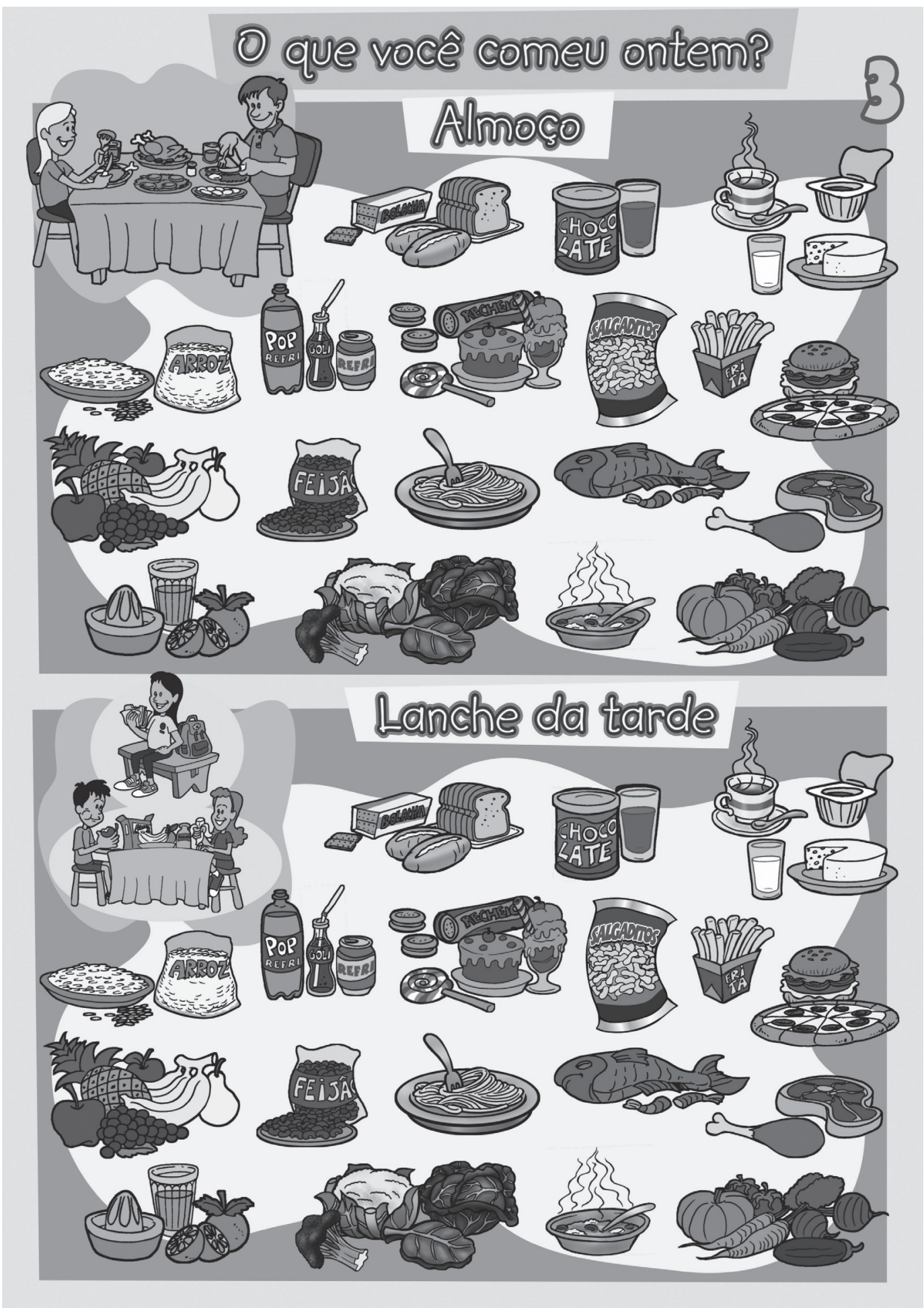

Fonte: Departamento de Nutrição, Universidade Federal de Santa Catarina. 


\section{Método de referência: observação}

O método de referência para testar a validade foi a observação direta dos alimentos consumidos em três refeições escolares (lanche da manhã, almoço e lanche da tarde), no dia anterior à aplicação do questionário.

A observação seguiu protocolo padronizado e foi realizada por pesquisadores e estudantes do Curso de Nutrição da Universidade do Vale do Itajaí, previamente treinados, conforme procedimentos já descritos 12. Os pesquisadores localizavam-se próximos às mesas onde eram feitas as refeições e ficavam responsáveis pela observação de, no máximo, cinco escolares, que eram identificados por meio de crachás com diferentes cores (cada cor correspondia a um observador). Os observadores anotavam os alimentos consumidos em um formulário para cada criança observada.

\section{Aplicação do QUADA}

Os alunos participantes do estudo foram agrupados de acordo com o ano escolar, em uma sala de aula, no dia seguinte à observação das refeições. O QUADA foi aplicado às turmas em forma de exercício dirigido, por um professor treinado, contando com o auxílio de dois pesquisadores que circulavam pela sala para esclarecer as dúvidas das crianças 13 .

Para a aplicação do questionário, foram utilizados quatro pôsteres $(90 \times 120 \mathrm{~cm})$ idênticos às páginas do questionário disponibilizado às crianças. Inicialmente, o professor apresentouse aos escolares, juntamente com seus auxiliares, explicou os objetivos de estarem reunidos naquela sala e enfatizou a importância de as crianças fornecerem respostas verdadeiras.

Os questionários foram distribuídos e os alunos, orientados a deixá-los sobre a mesa, prestando atenção somente às explicações do professor. As crianças foram situadas no tempo, lembrando do dia anterior e respondendo a perguntas do tipo: "Que dia da semana foi ontem?" e "Vocês vieram à escola no dia de ontem?”. Em seguida, por intermédio dos pôsteres, os alimentos do questionário foram identificados individualmente, em uma dinâmica participativa, sob a orientação do aplicador. Para cada alimento foram feitas perguntas do tipo: "Que alimento é este?" e “O que esta figura representa?". Ao término da identificação dos alimentos da parte superior do primeiro pôster, referente ao café da manhã, os alunos foram orientados a circular no seu questionário o que haviam consumido no dia de anterior (dia de ontem), durante a referida refeição.

Quando todos os escolares terminaram de assinalar os alimentos consumidos naquela re- feição, foram orientados a prestar atenção às explicações do professor sobre a refeição seguinte (lanche da manhã), localizada na parte inferior do pôster. O professor explicou que os alimentos eram os mesmos da parte superior e novamente reforçava a sua identificação, certificando-se de que os escolares reconheciam os alimentos apresentados. Ao término desta explicação, os escolares foram orientados a circularem no seu questionário o que haviam consumido no lanche da manhã do dia anterior.

Da mesma forma, mantendo rigorosamente o protocolo descrito, a aplicação foi conduzida para as demais refeições (almoço, lanche da tarde, jantar e lanche da noite). Os escolares foram orientados a não interferirem nas respostas dos colegas. Além disso, foram situados, reiteradamente, no tempo e na refeição em questão ("ainda estamos falando do lanche da manhã, que aconteceu no meio da manhã...”), sendo também relembrados de que não deveriam assinalar os alimentos caso a refeição em questão não tivesse sido feita ou se nenhum dos alimentos ou grupo de alimentos representados tivesse sido consumido.

Todas as refeições do dia anterior tiveram que ser relembradas e assinaladas no instrumento pelos alunos, mesmo aquelas que não foram observadas, pois o QUADA foi delineado com refeições ordenadas seqüencialmente, segundo o período do dia, para facilitar a lembrança dos alimentos consumidos no dia anterior e a aplicação pelo professor.

\section{Análises}

As análises foram conduzidas com os itens alimentares que apresentaram consumo relatado no QUADA-3 e observado. Os dados das hortaliças e verduras foram agrupados num mesmo item (hortaliças/verduras).

Para a validade externa do QUADA-3, foram calculados os valores de sensibilidade, especificidade, falsos-negativos e falsos-positivos, usando as observações como padrão-ouro ${ }^{20}$. Os resultados foram apresentados para as três refeições combinadas, considerando-se toda a amostra, estratificados por sexo, idade (agrupando-se os dados das crianças de 6 a 8 anos ou de 9 a 11 anos) e condição de excesso de peso (sim ou não). Para comparação com estudos similares, a estatística kappa também foi calculada, utilizando-se as referências de Landis \& Koch ${ }^{21}$ para interpretar os valores obtidos.

Adicionalmente, foram conduzidas análises de regressão logística multivariada para avaliar a influência de variáveis relacionadas aos participantes do estudo na discordância das respostas 
obtidas pelo QUADA-3 em relação à observação. A análise foi estratificada por refeição, tendo como variável dependente a discordância de respostas (sim/não) e, como variáveis independentes, o sexo (meninas/meninos), a idade (6 a 8 anos ou 9 a 11 anos) e o excesso de peso (não/sim).

O nível de significância adotado foi de 0,05 . As análises foram realizadas no programa SPSS, versão 13.0 (SPSS Inc., Chicago, Estados Unidos).

O estudo foi aprovado pelo Comitê de Ética em Pesquisas com Seres Humanos da Universidade Federal de Santa Catarina. Um termo de consentimento foi assinado pelos pais, e as crianças foram esclarecidas sobre a não-compulsoriedade do estudo.

\section{Resultados}

A amostra foi composta por 164 alunos (43,3\% meninas), dos quais 26 eram do segundo ano (53,8\% meninas), 37 do terceiro ( $54,1 \%$ meninas), 38 do quarto (39,4\% meninas) e 63 do quinto (34,9\% meninas). A idade média [ \pm desvio padrão (DP)] da amostra como um todo foi de 8,6 $\pm 1,3$ anos (meninas $=8,4 \pm 1,4$ anos; meninos $=8,8 \pm$ 1,3 anos), variando entre 6 a 11 anos para ambos os sexos. Os participantes do segundo e terceiro ano apresentaram idade média de 7,2 $\pm 0,6$ anos (meninas = 7,2 $\pm 0,7$ anos; meninos $=7,1 \pm 0,6$ ); já os escolares do quarto e quinto ano tinham idade média de 9,5 \pm 0,8 anos (meninas $=9,4 \pm$ 1,0 ; meninos $=9,5 \pm 0,7$ ). Dentre os participantes, $45(27,4 \%)$ apresentaram excesso de peso (17 meninas e 28 meninos) e uma criança de cada sexo tinha baixo peso para a idade.

Dos 21 alimentos apresentados na terceira versão do QUADA, 13 foram assinalados pelos observadores e recordados pelas crianças. Outros cinco foram reportados somente pelos alunos (sucos, leite, iogurte, salgadinho e batata frita) e três não foram consumidos nem observados (café com leite, queijo e hambúrguer/pizza). Para a amostra total, considerando-se todos os itens alimentares relatados e observados nas três refeições combinadas, o valor de sensibilidade sugere que a capacidade do QUADA-3 em detectar os alimentos realmente consumidos foi de $70,2 \%$. Por outro lado, a especificidade sugere que a capacidade do instrumento para indicar que não houve o consumo dos alimentos quando estes não foram realmente consumidos foi de $96,2 \%$. Cerca de um terço dos itens alimentares observados foi omitido pelos escolares (falsos-negativos) e somente $3,8 \%$ foram relatados no instrumento, mas não observados (intrusões ou falsos-positivos) (Tabela 1).

$\mathrm{Na}$ análise estratificada por item alimentar, os valores de sensibilidade foram superiores a $60 \%$ para a maioria dos grupos alimentares analisados (9/12), variando entre $67,1 \%$ (pão/bolacha salgada) a $97,5 \%$ (arroz). Conseqüentemente, as

Tabela 1

Valores de sensibilidade, especificidade, falsos-negativos, falsos-positivos e kappa da terceira versão do Questionário Alimentar do Dia Anterior (QUADA-3), por item alimentar em três refeições escolares combinadas, para todos os participantes (164 escolares $\times 3$ refeições $=492$ relatos/alimento). Balneário Camboriú, Santa Catarina, Brasil, 2007

\begin{tabular}{|c|c|c|c|c|c|c|c|}
\hline \multirow[t]{2}{*}{ Alimento } & \multicolumn{2}{|c|}{ Relato (\%) } & \multirow[t]{2}{*}{ Sensibilidade } & \multirow[t]{2}{*}{ Especificidade } & \multirow[t]{2}{*}{ Falsos-negativos } & \multirow[t]{2}{*}{ Falsos-positivos } & \multirow[t]{2}{*}{ Kappa * } \\
\hline & QUADA-3 & Observado & & & & & \\
\hline Pão/Bolacha salgada & 26,0 & 29,7 & $67,1(59,5-74,7)$ & $91,3(88,4-94,3)$ & $32,9(25,3-40,5)$ & $8,7(5,7-11,6)$ & $0,61(0,53-0,69)$ \\
\hline Bebida achocolatada & 25,6 & 22,4 & $73,6(65,4-81,9)$ & $88,2(85,0-91,5)$ & $26,4(18,1-34,6)$ & $11,8(8,5-15,0)$ & $0,59(0,50-0,68)$ \\
\hline Doces & 20,7 & 33,7 & $43,4(35,8-50,9)$ & $90,8(87,7-93,9)$ & $56,6(49,1-64,2)$ & $9,2(6,1-12,3)$ & $0,38(0,30-0,45)$ \\
\hline Arroz & 18,5 & 17,3 & $96,5(92,5-100,0)$ & $97,8(96,4-99,2)$ & $3,5(-0,4-7,5)$ & $2,2(0,8-3,6)$ & $0,92(0,86-0,98)$ \\
\hline Feijão & 14,6 & 12,4 & $88,5(80,5-96,5)$ & $95,8(93,9-97,7)$ & $11,5(3,5-19,5)$ & $4,2(2,3-6,1)$ & $0,78(0,68-0,89)$ \\
\hline Macarrão & 6,3 & 6,5 & $81,3(67,7-94,8)$ & $98,9(98,0-99,9)$ & $18,8(5,2-32,3)$ & $1,1(0,1-2,0)$ & $0,81(0,68-0,95)$ \\
\hline Peixe/Frutos do mar & 2,4 & 1,6 & $87,5(64,6-100,0)$ & $99,0(98,1-99,9)$ & $12,5(-10,4-35,4)$ & $1,0(0,1-1,9)$ & $0,69(0,37-1,01)$ \\
\hline Carne/Frango & 10,8 & 10,0 & $75,5(63,5-87,6)$ & $96,4(94,7-98,1)$ & $24,5(12,4-36,5)$ & $3,6(1,9-5,3)$ & $0,69(0,56-0,82)$ \\
\hline Sopa de verduras & 11,0 & 12,6 & $82,3(72,7-91,8)$ & $99,3(98,5-100,0)$ & $17,7(8,2-27,3)$ & $0,7(-0,1-1,5)$ & $0,86(0,78-0,95)$ \\
\hline Hortaliças/Verduras & 1,8 & 2,2 & $43,4(30,1-56,7)$ & $98,2(97,0-99,4)$ & $56,6(43,3-69,9)$ & $1,8(0,6-3,0)$ & $0,59(0,46-0,72)$ \\
\hline Frutas & 19,5 & 18,7 & $83,7(76,1-91,2)$ & $95,3(93,2-97,3)$ & $16,3(8,8-23,9)$ & $4,8(2,7-6,8)$ & $0,78(0,69-0,86)$ \\
\hline Refrigerantes & 2,4 & 5,1 & $36,0(17,2-54,8)$ & $99,4(98,6-100,0)$ & $64,0(45,2-82,8)$ & $0,6(-0,1-1,4)$ & $0,47(0,27-0,66)$ \\
\hline Todos & & & $70,2(67,2-73,2)$ & $96,2(95,7-96,7)$ & $29,8(26,8-32,8)$ & $3,8(3,3-4,3)$ & $0,69(0,66-0,72)$ \\
\hline
\end{tabular}

* Todos os valores de kappa foram significativos. 
taxas de falsos-negativos (omissões) foram mais baixas para estes alimentos. Os doces $(43,4 \%)$, hortaliças/verduras $(43,4 \%)$ e refrigerantes (36\%) apresentaram os menores valores de sensibilidade e, conseqüentemente, as maiores taxas de falsos-negativos (omissões). A especificidade foi alta para todos os alimentos, variando entre $99,4 \%$ (refrigerantes) a $88,2 \%$ (bebida achocolatada), gerando, por conseguinte, baixas taxas de falsos-positivos (intrusões) (Tabela 1).

De acordo com as referências recomendadas para a interpretação da estatística kappa 21, em média, obteve-se um valor classificado como substancial (k entre 0,61 e 0,80). Dos 12 itens alimentares analisados, três foram classificados como "quase perfeitos" $(\mathrm{k} \geq 0,81)$, seis foram "substanciais" (k entre 0,61 e 0,80 ), três foram moderados (kentre 0,41 e 0,60 ) e somente o grupo dos doces teve valor classificado como razoável (k entre 0,21 e 0,40 ) (Tabela 1).

A Tabela 2 apresenta os valores de sensibilidade e especificidade do QUADA-3, por item alimentar, estratificada segundo o sexo, a idade (6 a 8 anos ou 9 a 11 anos) e a condição de excesso de peso (não ou sim). Para alguns itens alimentares não foi possível realizar os testes estatísticos, em virtude do baixo consumo relatado e observado numa das categorias da variável estratificada. $\mathrm{Na}$ análise estratificada por sexo, faixa etária e excesso de peso, os valores de sensibilidade e especificidade para a maioria dos itens alimentares não apresentaram diferenças quando se considerou o intervalo de 95\% de confiança (IC95\%) (dados não apresentados). No entanto, os valores dos IC95\% indicaram maior sensibilidade para o relato de consumo de frutas entre as meninas; de doces entre os escolares de 9 a 11 anos; de macarrão entre os escolares que não apresentavam excesso de peso. Em média, os valores de sensibilidade foram ligeiramente mais elevados para meninas do que para os meninos e para os escolares que não apresentavam excesso de peso, quando comparados aos que apresentavam. Relativamente à idade, os valores de sensibilidade foram mais elevados para os escolares de 9 a 11 anos em comparação aos de 6 a 8 anos.

A análise de regressão logística multivariada (Tabela 3) mostra a taxa de discordância e os fatores associados a esta no que se refere à divergência de respostas entre o QUADA-3 e as observações, por refeição e item alimentar. A freqüência de discordância variou entre 3,7\% (peixe/frutos do mar no almoço) a 39,6\% (doces no lanche da manhã). A idade da criança foi o fator que mais influenciou na taxa de discordância. Os escolares de 6 a 8 anos apresentaram mais chance de discordância para os itens doces (lanche da manhã); feijão, carnes, hortaliças/verduras (al-

Valores de sensibilidade e especificidade da terceira versão do Questionário Alimentar do Dia Anterior (QUADA-3), por item alimentar em três refeições escolares combinadas, segundo o sexo, ano escolar e condição de excesso de peso *. Balneário Camboriú, Santa Catarina, Brasil, 2007.

\begin{tabular}{|c|c|c|c|c|c|c|c|c|c|c|c|c|}
\hline \multirow[t]{3}{*}{ Alimentos } & \multicolumn{4}{|c|}{ Sexo } & \multicolumn{4}{|c|}{ Idade (anos) } & \multicolumn{4}{|c|}{ Excesso de peso } \\
\hline & \multicolumn{2}{|c|}{ Meninos } & \multicolumn{2}{|c|}{ Meninas } & \multicolumn{2}{|c|}{$6-8$} & \multicolumn{2}{|c|}{$9-11$} & \multicolumn{2}{|c|}{ Não } & \multicolumn{2}{|c|}{ Sim } \\
\hline & $\mathrm{S}$ & $E$ & $\mathbf{S}$ & $E$ & $\mathrm{~S}$ & $E$ & $\mathrm{~S}$ & $E$ & S & $E$ & S & $E$ \\
\hline Pão/Bolacha salgada & 63,3 & 90,5 & 71,6 & 92,5 & 69,2 & 91,3 & 65,4 & 91,4 & 66,0 & 91,3 & 69,8 & 91,3 \\
\hline Bebida achocolatada & 67,6 & 86,6 & 84,6 & 90,2 & 66,7 & 84,6 & 83,0 & 90,9 & 80,6 & 89,1 & 60,5 & 85,6 \\
\hline Doces & 42,3 & 88,5 & 44,9 & 93,8 & 34,9 & 88,8 & 59,6 & 91,9 & 46,6 & 91,2 & 35,4 & 89,7 \\
\hline Arroz & 95,7 & 97,8 & 97,4 & 97,7 & 100 & 97,7 & 96,1 & 97,9 & 95,0 & 97,3 & 100,0 & 99,1 \\
\hline Feijão & 82,8 & 95,2 & 93,8 & 96,7 & 83,3 & 98,2 & 89,1 & 93,4 & 92,5 & 95,9 & 81,0 & 95,6 \\
\hline Macarrão & 80,0 & 98,1 & 83,3 & 100,0 & 75,0 & 99,1 & 83,3 & 98,8 & 76,9 & 98,8 & 100,0 & 99,2 \\
\hline Peixes/Frutos do mar & \multicolumn{2}{|c|}{$\mathrm{BC}$} & \multicolumn{2}{|c|}{$\mathrm{BC}$} & \multicolumn{2}{|c|}{$\mathrm{BC}$} & \multicolumn{2}{|c|}{$\mathrm{BC}$} & 87,5 & 98,5 & \multicolumn{2}{|c|}{$B C$} \\
\hline Carne/Frango & 70,8 & 95,7 & 80,0 & 97,3 & 71,4 & 99,1 & 76,2 & 93,8 & 79,4 & 96,3 & 66,7 & 96,7 \\
\hline Sopa de verduras & 82,9 & 99,2 & 81,0 & 99,5 & 98,2 & 94,4 & $\mathrm{BC}$ & 82,2 & 99,4 & 82,4 & 99,2 & \\
\hline Hortaliças/Verduras & \multicolumn{2}{|c|}{$B C$} & \multicolumn{2}{|c|}{$\mathrm{BC}$} & \multicolumn{2}{|c|}{$\mathrm{BC}$} & \multicolumn{2}{|c|}{$\mathrm{BC}$} & 18,2 & 98,0 & \multicolumn{2}{|c|}{$\mathrm{BC}$} \\
\hline Frutas & 75,0 & 96,0 & 95,0 & 94,2 & 72,2 & 96,1 & 86,5 & 94,3 & 83,3 & 94,7 & 85,0 & 96,5 \\
\hline Refrigerantes & 30,8 & 99,2 & 41,7 & 99,5 & \multicolumn{2}{|c|}{$\mathrm{BC}$} & 37,5 & 99,2 & \multicolumn{2}{|c|}{$\mathrm{BC}$} & 41,7 & 99,2 \\
\hline Todos & 67,2 & 95,6 & 74,6 & 97,1 & 61,4 & 96,6 & 76,6 & 95,9 & 72,3 & 96,2 & 65,9 & 96,3 \\
\hline
\end{tabular}

S: Sensibilidade; E: Especificidade; BC: baixo consumo relatado e observado (não foi possível realizar os testes estatísticos).

* Segundo os valores críticos do índice de massa corporal para de crianças e adolescentes brasileiros 15. 
Fatores associados à discordância de relatos de itens alimentares entre a terceira versão do Questionário Alimentar do Dia Anterior (QUADA-3) e as observações diretas, segundo a refeição escolar. Balneário Camboriú, Santa Catarina, Brasil, 2007 (N = 164).

\begin{tabular}{|c|c|c|c|c|}
\hline Refeição/Alimento & Discordância (\%) & $\begin{array}{c}\text { Sexo } \\
\text { Meninas * vs. Meninos } \\
\text { OR (IC95\%) }\end{array}$ & $\begin{array}{l}\text { Faixa etária (anos) } \\
9-11 \text { * vs. 6-8 } \\
\text { OR (IC95\%) }\end{array}$ & $\begin{array}{c}\text { Excesso de peso } \\
\text { Não * vs. Sim } \\
\text { OR (IC95\%) }\end{array}$ \\
\hline \multicolumn{5}{|l|}{ Lanche da manhã } \\
\hline Pão/Bolacha salgada & 28,0 & $0,66(0,32-1,35)$ & $1,21(0,60-2,44)$ & $0,75(0,34-1,65)$ \\
\hline Bebida achocolatada & 28,7 & $0,65(0,32-1,33)$ & $0,79(0,39-1,59)$ & $1,51(0,72-3,18)$ \\
\hline Doces & 39,6 & $1,08(0,51-2,29)$ & $0,09(0,04-0,20)$ & $2,19(0,95-5,02)$ \\
\hline \multicolumn{5}{|l|}{ Almoço } \\
\hline Arroz & 6,1 & $1,19(0,32-4,39)$ & $1,30(0,34-4,88)$ & $\mathrm{BD}$ \\
\hline Feijão & 14,0 & $0,53(0,21-1,39)$ & $3,98(1,37-11,55)$ & $1,47(0,56-3,86)$ \\
\hline Macarrão & 6,7 & $0,23(0,05-1,13)$ & $1,81(0,49-6,64)$ & $0,23(0,03-1,84)$ \\
\hline Carne/Frango & 16,5 & $0,44(0,18-1,11)$ & $7,27(2,33-22,70)$ & $1,40(0,55-3,57)$ \\
\hline Peixes/Frutos do mar & 3,7 & $0,20(0,02-1,77)$ & $5,39(0,60-48,02)$ & $0,49(0,05-4,42)$ \\
\hline Frutas & 4,9 & $0,18(0,02-1,48)$ & $0,57(0,13-2,55)$ & $0,32(0,04-2,69)$ \\
\hline Hortaliças/Verduras & 9,8 & $0,42(0,13-1,33)$ & $8,23(1,75-38,71)$ & $2,32(0,77-6,99)$ \\
\hline Sopa de verduras & 6,7 & $1,10(0,28-4,28)$ & $1,06(0,24-4,58)$ & $\mathrm{BD}$ \\
\hline \multicolumn{5}{|l|}{ Lanche da tarde } \\
\hline Pão/Bolacha salgada & 18,9 & $0,92(0,41-2,06)$ & $1,22(0,55-2,73)$ & $1,32(0,57-3,09)$ \\
\hline Bebida achocolatada & 14,6 & $0,55(0,19-1,58)$ & $0,17(0,06-0,50)$ & $4,56(1,72-12,08)$ \\
\hline Doces & 34,1 & $0,69(0,35-1,36)$ & $0,58(0,30-1,13)$ & $1,40(0,68-2,89)$ \\
\hline Refrigerante & 10,4 & $0,87(0,30-2,55)$ & $17,19(2,17-135,89)$ & $2,80(0,95-8,23)$ \\
\hline
\end{tabular}

BD: baixa discordância (não foi possível realizar a análise).

* Categoria de referência.

moço); pão/bolacha salgada e refrigerante/suco artificial (lanche da tarde). A condição de excesso de peso apresentou influência na discordância de respostas apenas para a bebida achocolatada no lanche da tarde. Não foram verificadas discordâncias de respostas entre os sexos, para todos os itens alimentares analisados.

\section{Discussão}

Este trabalho apresenta vários pontos positivos. O primeiro relaciona-se ao fato de terem sido adotados dois aspectos importantes no estudo de validação: o uso do mesmo período de consumo (três refeições escolares do dia anterior) para o método de referência e para o instrumento a ser validado, e o uso de uma medida de referência independente do questionário-teste e da memória para o auto-relato - a observação direta por pesquisadores. Apesar de diversos estudos terem usado o recordatório de 24 horas ou registros de alimentos para validar questionários breves de consumo de alimentos, estes métodos partilham das mesmas limitações, pois os erros associados ao método de ensaio e de referência não são independentes 4,5,6,8,9,22,23,24. O uso de observação direta como medida de referência vem ganhando importância por causa da qualidade das informações obtidas 1,2,7.

Outro aspecto positivo refere-se à análise de dados, conduzida para identificar o desempenho diagnóstico do instrumento e os erros provenientes de sub-relatos (omissões ou falsosnegativos) e super-relatos (intrusões ou falsospositivos), de acordo com o item alimentar, na amostra como um todo e estratificada por sexo e idade. Além disso, foram avaliados os fatores associados à discordância de respostas entre o QUADA-3 e as observações, segundo a refeição escolar. Os resultados gerados por estas análises fornecem importantes informações sobre os vieses de relato quando se aplicam questionários de consumo alimentar na faixa etária em estudo. Os vieses associados aos respondentes podem ter implicações nas avaliações de associações entre o consumo alimentar e desfechos de saúde e de programas de intervenção visando a mudanças de comportamento 24 . A ingestão diária de nutrientes, grupos de alimentos ou alimentos es- 
pecíficos tem sido considerada em estudos de validação de métodos de dietéticos em crianças e adolescentes 25,26 . No entanto, análises do padrão do consumo por refeição têm merecido menos atenção 25 .

O terceiro ponto positivo refere-se ao fato de o QUADA-3 ser resultado de sucessivos estudos de validação, que serviram para aprimorar o seu design e aperfeiçoar os respectivos protocolos, premissas também reforçadas em outros estudos 1,5,22. Deve-se ressaltar, ainda, que o QUADA foi desenvolvido considerando o estágio cognitivo das crianças de 7 a 10 anos de idade. A sua estrutura e o protocolo de aplicação permitem situar as crianças no tempo (dia de ontem), no espaço (refeições ordenadas cronologicamente) e estimular a imaginação visual (ilustração dos alimentos em cada uma das refeições), com o propósito de facilitar o processo de recuperação de informações. A imaginação visual e a familiaridade com o alimento são importantes mecanismos de recuperação de informações pela memória de curto prazo, utilizada por crianças em idade escolar 16,17,27.

Contudo, constataram-se, também, algumas limitações, as quais devem ser consideradas em futuras investigações. A primeira foi a utilização de uma amostra de conveniência de escolares do segundo ao quinto ano do Ensino Fundamental de uma escola pública, limitando a generalização dos resultados para diferentes contextos. Outra limitação foi que a observação deu-se apenas em dois lanches e uma refeição realizados na escola, não cobrindo, assim, todas as refeições do período de 24 horas. Numa próxima etapa, a validade do instrumento poderá ser testada com crianças da escola privada e nas refeições realizadas na casa dos escolares. Como terceira limitação, aponta-se o fato de o consumo alimentar ter sido obtido por meio do questionário, que inclui um dia de consumo ("O que você comeu ontem?"), o que fornece a medida da ingestão atual, apropriada para estimar a média de grupo ${ }^{28}$. Futuras pesquisas podem ser conduzidas para testar a validade do instrumento aplicado em múltiplos dias, uma vez que o instrumento é relativamente breve e fácil de aplicar no contexto escolar. Finalmente, o QUADA não foi delineado para estimar a ingestão de nutrientes, e questões sobre o tamanho das porções não foram incluídas. Esta última limitação se deve ao fato de o instrumento simplificar a tarefa da memória, focalizando a questão sobre os alimentos consumidos no dia anterior, evitando as dificuldades associadas à avaliação do tamanho da porção, o que o torna um questionário fácil de completar e de aplicar.

A comparação de resultados obtidos no presente estudo com os de outros questionários de avaliação de consumo alimentar é dificultada em face das diferenças de delineamento da investigação, de períodos e métodos de referência, de categorias de alimentos e métodos estatísticos empregados. A título de comparação, a Tabela 4 apresenta os valores de kappa obtidos para frutas e hortaliças nos estudos de validação do QUADA e de outros questionários para crianças em idade escolar. Os dados apresentados indicam que os valores da estatística kappa para frutas e verduras obtidos no QUADA-3 foram similares ou superiores aos obtidos em outros estudos (Tabela 4).

Constatou-se, na avaliação da sensibilidade e especificidade do QUADA-3, que os alimentos de consumo freqüente e ilustrados de forma isolada apresentaram os melhores parâmetros de desempenho diagnóstico para a validade. Esses alimentos parecem ter sido mais facilmente identificados no instrumento pelas crianças e codificados corretamente pelos pesquisadores. Por exemplo, arroz, feijão, frutas, sopa de verduras e macarrão apresentaram maiores percentuais de sensibilidade quando comparados às categorias que incluíam vários tipos de alimentos do mesmo grupo (doces, pães/bolacha salgada), ou que poderiam estar embutidos em preparações (hortaliças/legumes, doces no pão). Verificou-se, também, que a discordância de respostas entre o QUADA e as observações foi elevada para os doces no lanche da manhã e da tarde (Tabela 3). Dentre os possíveis motivos, cita-se que os observadores codificaram, no grupo dos doces, a geléia consumida com pães nessas refeições (dados observados no protocolo de aplicação); contudo, muitas crianças não circularam os doces nessas refeições, provavelmente porque a geléia não está ilustrada no QUADA-3. Crianças dessa faixa etária tendem a ser bastante literais, registrando ou recordando os alimentos claramente identificados por meio de ilustrações 22 . Destacase, pois, a importância do treinamento da equipe de observadores para codificarem corretamente os alimentos de acordo com a ilustração no questionário, o que, nesta avaliação, poderia ter produzido melhores resultados de validade para o grupo de doces.

Outra hipótese a ser considerada em relação ao sub-relato de consumo de doces é o fato de as crianças saberem que este item é socialmente indesejável de acordo com as recomendações nutricionais 28. Outros estudos de validação de questionários estruturados do dia anterior também reportaram menor precisão de informação para as categorias alimentares que incluíam vários tipos de alimentos ao invés de alimentos específicos (por exemplo, "verduras" em vez de "cenoura" ou "alface"; "doces" em vez de "sorvetes" ou "balas") 5,22. 
Valores da estatística kappa para frutas e verduras, observados em estudos de validação de questionários com grupos de alimentos, para crianças e adolescentes.

\begin{tabular}{|c|c|c|c|c|c|c|c|c|}
\hline Autores & País & $\mathbf{N}$ & $\begin{array}{l}\text { Idade } \\
\text { (anos) }\end{array}$ & $\begin{array}{c}\text { Questionário } \\
\text { validado }\end{array}$ & $\begin{array}{l}\text { Método de } \\
\text { referência }\end{array}$ & $\begin{array}{l}\text { Período de } \\
\text { referência }\end{array}$ & Frutas & Verduras \\
\hline Presente estudo & & 164 & $7-10$ & QUADA-3 & Observação & $\begin{array}{c}3 \text { refeições } \\
\text { escolares }\end{array}$ & 0,78 & $\begin{array}{c}\text { 0,86 (sopa); } \\
\text { 0,59 (hortaliças) }\end{array}$ \\
\hline Assis et al. 12 & Brasil & 131 & $8-10$ & QUADA-2 & Observação & $\begin{array}{c}3 \text { refeições } \\
\text { escolares }\end{array}$ & 0,76 & 0,71 \\
\hline Barros et al. 10 & Brasil & 69 & 1a-4a * & DAFA & $\begin{array}{c}\text { Recordatório } \\
24 \text { horas }\end{array}$ & 24 horas & $0,19-0,48$ ** & $0,84-0,48 * \star$ \\
\hline Koehler et al. 4 & Estados Unidos & 120 & $10-14$ & $\begin{array}{l}\text { Recordatório } 24 \\
\text { horas/estruturado }\end{array}$ & $\begin{array}{l}\text { Recordatório } \\
24 \text { horas }\end{array}$ & 24 horas & 0,29 & 0,28 \\
\hline Hoelscher et al. 22 & Estados Unidos & 209 & $8 \underline{a} / 11 \underline{a}$ * & $\begin{array}{l}\text { Recordatório } 24 \\
\text { horas/estruturado }\end{array}$ & $\begin{array}{c}\text { Recordatório } \\
24 \text { horas }\end{array}$ & 24 horas & 0,33 & 0,32 \\
\hline $\begin{array}{l}\text { Thiagarajah } \\
\text { et al. } 23\end{array}$ & Estados Unidos & 121 & $9-11$ & $\begin{array}{l}\text { Recordatório } 24 \\
\text { horas/estruturado }\end{array}$ & $\begin{array}{c}\text { Recordatório } \\
24 \text { horas }\end{array}$ & 24 horas & 0,27 & 0,17 \\
\hline Edmunds et al. 2 & Inglaterra & 255 & $7-9$ & $\begin{array}{l}\text { Recordatório } 24 \\
\text { horas/estruturado }\end{array}$ & Observação & Lanches escolares & \multicolumn{2}{|c|}{$0,54 * \star \star ; 0,58 \#$} \\
\hline Moore et al. 8 & Inglaterra & 78 & $9-11$ & $\begin{array}{l}\text { Recordatório } 24 \\
\text { horas/computador }\end{array}$ & $\begin{array}{c}\text { Recordatório } \\
24 \text { horas }\end{array}$ & $\begin{array}{c}2 \text { refeições } \\
\text { escolares }\end{array}$ & 0,29 & NA \\
\hline Moore et al. 6 & Inglaterra & 374 & $9-11$ & $\begin{array}{l}\text { Recordatório } 24 \\
\text { horas/estruturado }\end{array}$ & $\begin{array}{l}\text { Recordatório } \\
24 \text { horas }\end{array}$ & Almoço & 0,42 & 0,38 \\
\hline Vereecken et al. 9 & Bélgica & 136 & $12-14$ & $\begin{array}{l}\text { Recordatório } 24 \\
\text { horas/computador }\end{array}$ & Registro & Seis refeições & 0,71 & 0,76 \\
\hline Vereecken et al. 9 & Bélgica & 101 & $11-14$ & $\begin{array}{l}\text { Recordatório } 24 \\
\text { horas/computador }\end{array}$ & $\begin{array}{l}\text { Recordatório } \\
24 \text { horas }\end{array}$ & Seis refeições & 0,57 & 0,81 \\
\hline
\end{tabular}

QUADA: Questionário Alimentar do Dia Anterior; DAFA: Dia Típico de Atividade Física e de Consumo Alimentar; NA: não analisado.

* Série escolar.

** Valores para o consumo no período da manhã e da noite.

*** Primeira rodada de coleta de dados: resultados para frutas e verduras combinados.

\# Segunda rodada de coleta de dados: resultados para frutas e verduras combinados.

No que concerne a intrusões e omissões, cabe ressaltar que a taxa de falsos-positivos (intrusões) foi relativamente baixa. Para os falsos-negativos (omissões), as maiores taxas foram encontradas para os refrigerantes, doces e legumes/hortaliças. (Tabela 1). Estudos que avaliaram a acurácia de recordatórios de 24 horas contra as observações das refeições de escolares americanos da quarta série mostraram que, mesmo quando as entrevistas foram conduzidas no final do dia em que o consumo foi observado, as crianças omitiram um terço dos alimentos observados (café da manhã e almoço); além disso, quase um quinto dos itens que as crianças relataram consumir nas duas refeições não foi observado ${ }^{3}$. Em pesquisa conduzida para a validação de um questionário de consumo de alimentos em cinco períodos do dia, contra registros alimentares, os doces foram os alimentos mais omitidos, com taxas de omissão variando de $9 \%$ para o lanche da manhã a $27 \%$ no almoço 6 .

No presente estudo, a idade foi a variável que mais influenciou a discordância das respostas das crianças em relação às anotações dos observadores. Alunos de 6 a 8 anos apresentaram mais respostas discordantes em relação aos de 9 a 11 anos. O nível de desenvolvimento cognitivo das crianças de 9 a 11 anos (vocabulário mais desenvolvido em relação ao nome de alimentos e capacidade de abstração) pode ter contribuído para a melhor concordância das respostas, em relação aos escolares mais novos. Também nos Estados Unidos, dois estudos conduzidos para validar questionários de freqüência alimentar contra múltiplos recordatórios de 24 horas apresenta- 
ram resultados de correlações mais fortes para os escolares com mais idade, em comparação aos mais novos ${ }^{29}$, e para crianças que freqüentavam a sexta e a sétima série, em comparação àquelas que estavam na quarta e na quinta série 30 . Isso indica que níveis de alfabetização e maturidade são importantes fatores a se considerar no delineamento de questionários de consumo alimentar.

Os dados encontrados indicaram que o sexo não influenciou na discordância dos resultados, e que o excesso de peso esteve associado à discordância somente para o item bebida achocolatada. Todavia, o tamanho da amostra pode ter sido pequeno para detectar possíveis diferenças e generalizar os resultados. Baxter et al. ${ }^{3}$ também não encontraram diferença significativa entre os sexos no que concerne à acurácia dos relatos auto-respondidos por entrevistas recordatórias validadas, contra as observações escolares. Em outro estudo, os autores 31 relataram que crianças com IMC mais elevado consumiram e omitiram mais quilocalorias, quando comparadas àquelas com IMC mais baixo.

Portanto, os resultados deste estudo mostraram que a terceira versão do QUADA pode gerar dados válidos para a maioria dos alimentos ilustrados no questionário, quando aplicada junto a uma amostra de escolares do segundo ao quinto ano do ensino fundamental da escola pública.

\section{Resumo}

O estudo objetivou conferir validade à terceira versão do Questionário Alimentar do Dia Anterior (QUADA3) para escolares. $O Q U A D A-3$ é um instrumento estruturado com seis refeições e 21 alimentos. Participaram 164 escolares de 6 a 11 anos de idade, de uma escola pública de período integral, em 2007. A validade foi testada pela comparação entre os itens alimentares selecionados no questionário e a observação direta de alimentos consumidos em três refeições escolares do dia anterior (lanche da manhã, almoço, lanche da tarde). Análise de regressão logística multivariada foi conduzida para avaliar a discordância entre as respostas do questionário e o registro das observações. Os resultados indicaram alta sensibilidade e especificidade para a maioria dos itens alimentares. Em média, os valores de sensibilidade e especificidade foram de 70,2\% e de 96,2\%, respectivamente, para os 12 itens alimentares nas três refeições combinadas. Os escolares de 6 a 8 anos, quando comparados aos de 9 a 11 anos, apresentam maiores taxas de discordância de respostas entre o QUADA-3 e as observações. Os resultados indicaram que o QUADA-3 pode gerar dados válidos para avaliar o consumo alimentar do dia anterior em nível de grupo de escolares.

Consumo de Alimentos; Questionários; Criança

\section{Colaboradores}

M. A. A. Assis, J. Benedet, R. Kerpel, F. A. G. Vasconcelos e P. F. Di Pietro delinearam o estudo. R. Kerpel participou da coleta de dados. E. Kupek e M. A. A. Assis realizaram a análise de dados. Todos os autores participaram da redação final do artigo.

\section{Agradecimentos}

Aos professores, pais e alunos do Centro Integrado de Educação Pública (CIEP) Rodesindo Pavan (Balneário Camboriú). À professora Dayse Dietrich de Athayde, do Apoio Pedagógico Especial do CIEP Rodesindo Pavan, pela aplicação do QUADA-3. Às nutricionistas Débora Guimarães, Fabiana Martin Baramarchi e Mariene Casanova, pelo auxílio na coleta de dados. Agradecemos ao Conselho Nacional de Desenvolvimento Científico e Tecnológico pelo financiamento do projeto $(\mathrm{CNPq}$, processo $n^{\circ}$. 402322/2005-3).

\section{Referências}

1. Baxter SD. Cognitive processes in children's dietary recalls: insight from methodological studies. Eur J Clin Nutr 2009; 63 Suppl 1:S19-32.

2. Edmunds LD, Ziebland S. Development and validation of the day in the life questionnaire (DILQ) as a measure of fruit and vegetable questionnaire for 7-9 year olds. Health Educ Res 2002; 17:211-20.

3. Baxter SD, Thompson WO, Davis HC, Johnson MH. Impact of gender, ethnicity, meal component, and time interval between eating and reporting on accuracy of fourth-graders' self-reports of school lunch. J Am Diet Assoc 1997; 97:1293-8. 
4. Koehler KM, Cunningham-Sabo L, Lambert LC, McCalman R, Skipper BJ, Davis SM. Assessing food selection in a health promotion program: validation of a brief instrument for American Indian children in the Southwest United States. J Am Diet Assoc 2000; 100:205-11.

5. Andersen LF, Bere E, Kolbjornsen N, Klepp K-I. Validity and reproducibility of self-reported intake of fruit and vegetable among 6th graders. Eur J Clin Nutr 2004; 58:771-7.

6. Moore GF, Tapper K, Murphy S, Clark R, Lynch R, Moore L. Validation of a self-completion measure of breakfast foods, snacks and fruits and vegetables consumed by 9 - to 11-year-old schoolchildren. Eur J Clin Nutr 2007; 61:420-30.

7. Baranowski T, Islam N, Baranowski J, Cullen KW, Myres D, Marsh T, et al. The food intake recording software system is valid among fourth-grade children. J Am Diet Assoc 2002; 102:380-5

8. Moore L, Tapper K, Dennehy A, Cooper A. Development and testing of a computerised $24 \mathrm{~h}$ recall questionnaire measuring fruit and snack consumption among 9-11 year olds. Eur J Clin Nutr 2005; 59:809-16.

9. Vereecken CA, Covents M, Matthys C, Maes L. Young adolescents' nutrition assessment on computer (YANA-C). Eur J Clin Nutr 2005; 59:658-67.

10. Barros MVG, Assis MAA, Pires MC, Grossemann S, Vasconcelos FAG, Luna, MEP, et al. Validity of physical activity and food consumption questionnaire for children aged seven to ten years old. Rev Bras Saúde Matern Infant 2007; 7:437-48.

11. Lobo AS, Assis MAA, Barros MVG, Calvo MCM, Freitas SFT. Reprodutibilidade de um questionário de consumo alimentar para crianças em idade escolar. Rev Bras Saúde Matern Infant 2008; 8:55-63.

12. Assis MAA, Guimarães D, Calvo MCM, Barros MVG, Kupek E. Reprodutibilidade e validade de questionário de consumo alimentar para escolares. Rev Saúde Pública 2007; 41:1054-7.

13. Assis MAA, Kupek E Guimarães D, Calvo MCM, Andrade DF, Bellisle F. Test-retest reliability and external validity of the Previous Day Food Questionnaire for 7- to 10-year-old school children. Appetite 2008; 51:187-93

14. World Health Organization. Physical status: the use and interpretation of anthropometry. Geneva: World Health Organization; 1995. (Technical Report Series, 854)

15. Conde WL, Monteiro CA. Body mass index cutoff points for evaluation of nutritional status in Brazilian children and adolescents. J Pediatr (Rio J) 2006; 82:266-72.

16. Baranowski T, Domel SB. A cognitive model of children's reporting of food intake. Am J Clin Nutr 1994; 59 Suppl:212S-7S.

17. Foster E, Adamson AJ, Anderson AS, Barton KL, Wrieden WL. Estimation of portion size in children's dietary assessment: lessons learnt. Eur J Clin Nutr 2009; 63:S45-9.
18. Weis B, Chaim NA, Belik W. Manual de gestão eficiente da merenda escolar. http://www.acao fomezero.org.br/arquivos/ManualdeGestaoEfici entedaMerendaEscolar2005.pdf (acessado em 10/ Set/2006).

19. Ministério da Saúde. Guia alimentar para a população brasileira. http://www.saude.gov.br (acessado em 10/Set/2006).

20. Szklo M, Nieto FJ. Epidemiology. Beyond the basics. Sudbury: Jones and Bartlett Publishers; 2004.

21. Landis JR, Koch GG. The measurement of observer agreement for categorical data. Biometrics 1977; 33:159-74.

22. Hoelscher DM, Day RS, Kelder SH, Ward JL. Reproducibility and validity of de secondary level school-based nutrition monitoring student questionnaire. J Am Diet Assoc 2003; 103:186-94.

23. Thiagarajah K, Fly AD, Hoelscher DM, Bai Y, Lo K, Leone A, et al. Validating the food behavior questions from the elementary school SPAN questionnaire. J Nutr Educ Behav 2008; 40:305-10.

24. Moore GF, Tapper K, Moore L, Murphy S. Cognitive, behavioral, and social factors are associated with bias in dietary questionnaire self-reports by schoolchildren aged 9 to 11 years. J Am Diet Assoc 2008; 108:1865-73.

25. Sjöberg A, Hulthén L. Assessment of habitual meal pattern and intake of foods, energy and nutrients in Swedish adolescent girls: comparison of diet history with 7-day record. Eur J Clin Nutr 2004; 58:1181-9.

26. Voci SM, Enes CC, Slater B. Validação do Questionário de Freqüência Alimentar para Adolescentes (QFAA) por grupos de alimentos em uma população de escolares. Rev Bras Epidemiol 2008; 11: 561-72.

27. Baxter SD, Thompson WO, Davis HC, Johnson M. How do you remember you ate...? A Delphi technique study to identify retrieval categories from fourth-grade children. J Am Diet Assoc 1997; 97: 31-6.

28. Willet WC. Nutritional epidemiology. 2nd Ed. Oxford: Oxford University Press; 1998.

29. Rockett HR, Breitenbach M, Frazier AL, Witschi J, Wolf AM, Field AE, et al. Validation of a youth/adolescent food frequency questionnaire. Prev Med 1997; 26:808-16.

30. Field AE, Peterson KE, Gortmaker SL, Cheung L, Rockett H, Fox MK, et al. Reproducibility and validity of a food frequency questionnaire among fourth to seventh grade inner-city school children: implications of age and day-to-day variation in dietary intake. Public Health Nutr 1999; 2:293-300.

31. Baxter SD, Smith AF, Nichols MD, Guinn CH, Hardin JW. Children's dietary reporting accuracy over multiple 24 -hour recalls varies by body mass index category. Nutr Res 2006; 26:241-8.

Recebido em 15/Jul/2008

Versão final reapresentada em 28/Abr/2009 Aprovado em 04/Mai/2009 